\title{
Welcome to the year 2016
}

\author{
Nadia Magnenat-Thalmann ${ }^{1}$
}

Published online: 29 January 2016

(C) Springer-Verlag Berlin Heidelberg 2016

Welcome to 2016. I wish you a very active and fruitful academic year.

The Visual Computer Journal is one of the most successful journals in the world. We have received during the year 2015 more than 600 submissions. After careful review by at least 3 reviewers, 132 papers have been accepted. The Visual Computer publishes 12 issues a year, each containing around 10 papers. The acceptance rate is about 20 per cent. As we can see, it is an incredible number of papers we have to deal with and I take this opportunity to thank our associate editors and reviewers for their tremendous work.

Throughout 2015, some associate editors left the Editorial Board. I would like to thank them for their great contribution to The Visual Computer:

- Paolo Cignoni, ISTI-CNR, Italy

- Eddy Flerackers, University of Hasselt, Belgium

- Shi-Min Hu, Tsinghua University, China

- Tong-Yee Lee, National Cheng-Kung University, Taiwan

- Niloy J. Mitra, UCL

- Philip Ogunbona, University of Wollongong, Australia

- Olga Sorkine, ETH, Switzerland

I am happy to welcome three new associate editors-in-chief:

- Hui Huang, Shenzen Institute of Advanced Technology, China

- Daniele Panozzo, New York University, USA

Nadia Magnenat-Thalmann

thalmann@miralab.ch

1 MIRALab-CUI, University of Geneva, Battelle, Building A,

7, Route de Drize, 1227 Carouge, Geneva, Switzerland
- Hyewon Seo, CNRS and University of Strasbourg, France

I would also like to welcome the new Associate Editors who have joined the editorial board this year:

- Frederic Cordier, University of Haute Alsace and University of Strasbourg, France

- Rongrong Ji, Xiamen University, China

- Liang Lin, Sun Yat-sen University, Guangzhou, China

- Kevin Xu, National University of Defense Technology, China

- Junfeng Yao, Xiamen University, China

- Kang Zhang, University of Texas at Dallas, USA

In 2016, the journal will publish the best of papers of four conferences: CGI 2016, Cyberworlds, VSGAmes and 3DOR.

Thank you to the Editorial Assistants, Aryel Beck and Jan Perhac for their help and support.

I would also like to thank our Editorial Director at Springer, Beverley Ford, and Rachel Roberts, the Editor of the journal for their support and commitment. Thank you also to Beate Uhl, the Production Editor, and to Sneha Rahul for their tremendous work and constant assistance.

Nadia Magnenat Thalmann

Editor-in-Chief 


\section{Reviewers List}

Adabala Neeharika

Agus Marco

Akgul Ceyhun

Akgül Yusuf Sinan

Akyuz Ahmet

Allen Brian

Alsallakh Bilal

Anbarjafari Gholamreza

Ando Ryoichi

Arora Chetan

Artusi Alessandro

Attene Marco

Au Chi Kit

Auzinger Thomas

Avril Quentin

Baciu George

Baek Nakhoon

Balcisoy Selim

Banterle Francesco

Barla Pascal

Barranco Francisco

Barthe Loic

Baumann Florian

Ben Hamza Abdessamad

Berard Pascal

Berger Kai

Berretti Stefano

Berseth Glen

Bian Zhenpeng

Biasotti Silvia

Blanes Ian

Bleyer Michael

Bogomjakov Alexander

Bonneau Georges-Pierre

Bonneel Nicolas

Bottoni Paolo

Bouaziz Sofien

Boulanger Pierre

Boulic Ronan

Bredif Mathieu

Bresson Xavier

Bronson Jonathan

Brunton Alan
Buchmann Dipl.-Math. Rasmus

Bühler Katja

Bulbul Abdullah

Callet Patrick

Cao Chen

Capin Tolga

Cerri Andrea

Changxi Zheng

Charalambous Panayiotis

Chattopadhyay Debaleena

Chen Tao

Chen Xiaobai

Chen Xuejin

Chen Soong-Der

Chen Zhonggui

Chen David

Chen Wei

Chen Renjie

Chen Liming

Cheng Ming-Ming

Cheng Erkang

Cheng Qiang

Chi-Fu William, Lai

Chover Miguel

Christensen Niels

Chu Hung-Kuo

Chu Nelson

Chuang Yung-Yu

Chung Haeyong Chung

Cong Yang

Constable Martin

Conte Donatello

Cordier Frederic

Cueto Elias

Cui Weiwei

Dai Yuchao

De-Maeztu Leonardo

Derpanis Konstantinos

Dick Christian

Ding Yuanyuan

Dong Weiming

Du Peng

Du Song-Pei

Duriez Chirstian
Dutagaci Helin

Duvenhage Bernardt

Dyer Ramsay

Ecins Aleksandrs

Edwards Essex

Einecke Nils

El-Sana Jihad

Fan Bin

Fang Shuai

Favorskaya Margarita

Feito Francisco

Feixas Miquel Feixas

Ferreira Nivan

Franco Annalisa

Franklin W. Randolph

Fu Hongbo

Fusco Giovanni

Ganovelli Fabio

Gao Lin

Gao Yi

Gao Xinbo

Gasteiger Rocco

Gehrig Stefan

Geng Weidong

Ghanem Bernard

Ghorbel Faouzi

Ghosh Abhijeet

Giorgi Daniela

Gorthi Subrahmanyam

Goshtasby Arthur

Gu Jinwei

Guennebaud Gael

Guo Xiaohu

Guo Yanwen

Ha Linh

Hadwiger Markus

Haines Eric

Hall Peter

Hanming Huang

Haworth M. Brandon

He Lei

Herout Adam

Ho Edmond

Hoang Thuc 


\begin{tabular}{|c|c|c|}
\hline Ho-Hyoung Choi & Kang Soo Min & Li Lei \\
\hline Hong Xiaopeng & Kang Cuicui & Li Guiqing \\
\hline Hong Wien & Kang Xudong & Li Hongsong \\
\hline Hotz Ingrid & Kapadia Mubbasir & Li Jia \\
\hline Hou Qiming & Kim Vladimir & Li Yusha \\
\hline Hou Junhui & Kim Chang-Hun & Li Xin \\
\hline Hoyet Ludovic & Kim Jinman & Li Xin \\
\hline Hu Zhe & Kim Tae-Joon & $\mathrm{Li} \mathrm{Yi}$ \\
\hline Hu Xinghong & Kiselev Victor & Li Wanqing \\
\hline Hu Jun & Klein Maximilian & Lian Zhouhui \\
\hline Hu Shi-Min & Kolingerova Ivana & Liang Hui \\
\hline Huang Xiaolei & Koo Bongjin & Liang Xiaodan \\
\hline Huang Jin & Koo Sang Ok & Liao Jing \\
\hline Huang Jia-Bin & Kosinka Jiri & Lima João Paulo \\
\hline Huang Hui & Kuang Zhanghui & Lin Haiting \\
\hline Huang Lei & Kumar Ashnil & Lin Feng \\
\hline Huang Di & Kumar Dhananjay & Lin Hongwei \\
\hline Huang Sheng Jun & Kumar Subodh & Lin Hsueh-Yi \\
\hline Huang Shi-Sheng & Kwan Kin Chung & Lin Chia-Chen \\
\hline Hullin Matthias & Kwok Tsz Ho & Ling Haibin \\
\hline Hulusic Vedad & Kwok Ngai Ming & Liu Yong-Jin \\
\hline Hwang Wen-Liang & Kwon Taesoo & Liu Ligang \\
\hline Ihmsen Markus & Laffont Pierre-Yves & Liu Zhi \\
\hline Inselberg Alfred & Laga Hamid & Liu Xueting \\
\hline Irfan Mohammad & Lai Yu-Kun & Liu Yonghuai \\
\hline Jacob Wenzel & Lai Yu-Chi & Liu Chunxiao \\
\hline Jaroslav krivanek & Lai Shang-Hong & Ljung Patric \\
\hline Ji Yu & Lan Tian & Lopez Luis \\
\hline Ji Hui & Lancelle Marcel & Lou Yifei \\
\hline Jiang Nianjuan & Lanz Oswald & Lu Yongtao \\
\hline Jiang Xudong & Lau Manfred & Lu Cewu \\
\hline Jiang Fan & Le Binh & Lu Aidong \\
\hline Jin Xiaogang & Le Meur Olivier & Lucas Yves \\
\hline Jing Wu & Lee Sung Hee & Lun Daniel Pak-Kong \\
\hline Jing Xuan & Lee Sungkil & Luo Ye \\
\hline Jones Ben & Lee Hwanyong & Ma Ziyang \\
\hline Jun Lee & Lee Hasup & Ma Ziyang \\
\hline Jung Younhyun & Lee In-Kwon & Maciel Anderson \\
\hline Jung Claudio & Lee Yunjin & Maddock Steve \\
\hline Junhui Hou & Lemercier Samuel & Makihara Yasushi \\
\hline Kakarala Ramakrishna & Li Jianmin & Mao Xiaoyang \\
\hline Kallmann Marcelo & Li Guorong & Mao Ren \\
\hline Kalra Prem & Li Chenfeng & Mao Tianlu \\
\hline Kaneda Kazufumi & Li Xian-Ying & Margolin Ran \\
\hline
\end{tabular}




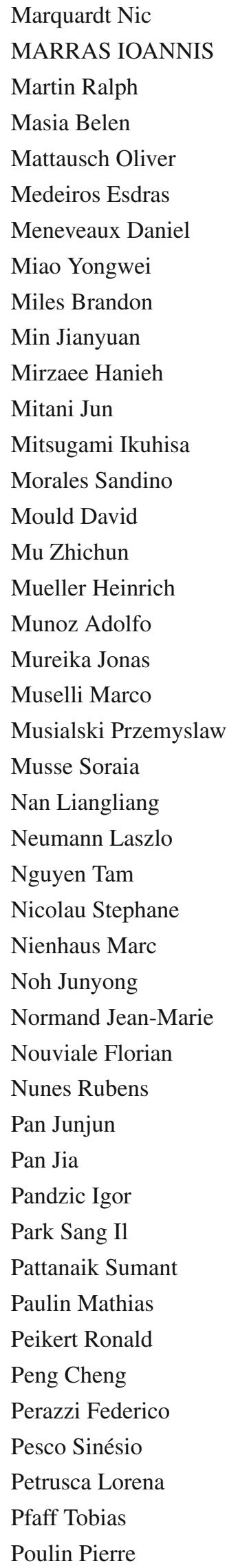

Prasad Mukta

prazak Martin

Preim Bernhard

Pronost Nicolas

PV Hareesh

Qi Xianbiao

Raman Shanmuganathan

Raman Shanmuganathan

Rautek Peter

Razdan Anshuman

Reiterer Harald

Ren Jianfeng

Ren Zhong

Rezk Salama Christof

Ribeiro Pedro Canotilho

Rokita Przemyslaw

Rosin Paul

Rückert Ulrich

S. Morse Bryan

Sadlo Filip

SanMiguel Juan Carlos

Schaefer Scott

Schein Sagi

Schein Sagi

Seo Hae Jong

Serra-Sagrista Joan

Shan Shiguang

Shao Tianjia

Shen Jianbing

Shen Wei

Shen Xiaohui

Sheng Bin

Sheng Yun

Shi Conglei

Shi Jianping

Shin Hyun Joon

Sideris Costas

Siebert Paul

Simon Gilles

Sitthi-amorn Pitchaya

Song Xuan

Song Byung Cheol

Song Ran

Sourimant Gael
Spetsakis Minas

Stolpner Svetlana

Sumengen Selcuk

Sun Xin

Sun Xianfang

Suter Susanne

Szirmay-Kalos Laszlo

Tai Yu-Wing

Tan Xiaoyang

Tang Li-an

Tang Cheng-Yuan

Tang Min

Tarnowsky Andreas

Thalmann Daniel

Thielhelm Hannes

Timofte Radu

Tisserand Yvain

Tong Ruofeng

Tong Yiying

Toth Robert

Tsai Piyu

Ugail Hassan

Uhlmann Virginie

Vais Alexander

van Kaick Oliver

Vandeborre Jean-Philippe

Vanek Juraj

Veltkamp Remco

Vergne Romain

Viola Ivan

Vlasov Roman

von Wangenheim Aldo

Vouga Etienne

Wachs Juan

Walker James S.

Walter Marcelo

Wan Jing

Wang Rui

Wang Yu-Shuen

Wang Junqiu

Wang Fan

Wang Chaohui

wang guoping

Wang Yangang 
Wang Yilun

Wang Zeng-Fu

Wang Zhaoqi

Wang Jiang

Wang Lei

Wang Le

Wang Lili

Wegert Elias

Weinmann Michael

Weissman Steffen

Wessel Raoul

Westermann Rüdiger

Winnemoller Holger

Wischgoll Thomas

Wloka Calden

Wolkowicz Jacek

Wolter Franz - Erich

Wu Jun

Wu Chenglei

Wu Hongzhi

Wu Chunlin

Wu Yi

$\mathrm{Wu} \mathrm{Yi}$

Xia Jiazhi

Xia Yong

Xiao Chunxia
Xin Shiqing

Xu Kai

Xu Kun

$\mathrm{Xu} \mathrm{Li}$

Xu Mingliang

Yan Dong-Ming

Yang Yong-Liang

Yang Bo

Yang Xiao

Yang Jianchao

Yang Xue Dong

Yang Yezhou

Yang Tao

Ye Jinwei

Yin Xiaotian

Yoon Ju Hong

Yoon Jungho

Yoon Sung-Eui

You Lihua

Yuan Xiaoru

Yuksel Cem

Zhang Fang-Lue

Zhang Lei

Zhang Yun

Zhang Zhuming

Zhang Song-Hai
Zhang Tianzhu

Zhang Junping

Zhang Haichao

Zhang Jianmin

Zhang Xinyu

Zhang Eugene

Zhang Lihe

Zhang Shaoting

Zhao Xuemei

Zhao Guoying

Zhao Chen

Zheng Youyi

Zheng Changwen

Zheng Jianmin

Zhong Fan

Zhou Shizhe

Zhou Boleh

Zhou Hongbo

Zhou Qian-Yi

Zhu Hai Chao

Zhu Lifeng

Zhu Hongyuan

Zou Changqing

Zsolnai Karoly 